\title{
Cluster observations of near-Earth magnetospheric lobe plasma densities - a statistical study
}

\author{
K. R. Svenes ${ }^{1}$, B. Lybekk ${ }^{2}$, A. Pedersen ${ }^{2}$, and S. Haaland ${ }^{3,4}$ \\ ${ }^{1}$ Norwegian Defence Research Establishment, P.O. Box 25, 2027 Kjeller, Norway \\ ${ }^{2}$ University of Oslo, Department of Physics, Norway \\ ${ }^{3}$ University of Bergen, Department of Physics, Norway \\ ${ }^{4}$ Max-Planck Institute for Solar System Research, Lindau, Germany
}

Received: 7 May 2008 - Revised: 9 July 2008 - Accepted: 22 August 2008 - Published: 22 September 2008

\begin{abstract}
The Cluster-mission has enabled a study of the near-Earth magnetospheric lobes throughout the waning part of solar cycle 23. During the first seven years of the mission the satellites crossed this region of space regularly from about July to October. We have obtained new and more accurate plasma densities in this region based on spacecraft potential measurements from the EFW-instrument. The plasma density measurements are found by converting the potential measurements using a functional relationship between these two parameters. Our observations have shown that throughout this period a full two thirds of the measurements were contained in the range $0.007-0.092 \mathrm{~cm}^{-3}$ irrespective of solar wind conditions or geomagnetic activity. In fact, the most probable density encountered was $0.047 \mathrm{~cm}^{-3}$, staying roughly constant throughout the entire observation period. The plasma population in this region seems to reflect an equilibrium situation in which the density is independent of the solar wind condition or geomagnetic activity. However, the high density tail of the population $\left(n_{e}>0.2 \mathrm{~cm}^{-3}\right)$ seemed to decrease with the waning solar cycle. This points to a source region influenced by the diminishing solar UV/EUVintensity. Noting that the quiet time polar wind has just such a development and that it is magnetically coupled to the lobes, it seems likely to assume that this is a prominent source for the lobe plasma.
\end{abstract}

Keywords. Magnetospheric physics (Polar cap phenomena; Solar wind-magnetosphere interactions; Instruments and techniques)

\section{Introduction}

The four Cluster spacecraft (see Escoubet et al., 1997a) have now been in orbit for more than seven years, providing a

Correspondence to: K. R. Svenes

(ksv@ffi.no) wealth of measurements on various aspects of the interaction between the solar wind and the magnetosphere. Consequently, there are now available adequate data sets to carry out specialized studies on particular topics within this framework. In that respect, it should also be noted that this time period coincides with the waning phase of solar cycle 23 providing measurements over a range of magnetospheric conditions.

In the present paper we will discuss plasma density measurements obtained during traversals of the magnetospheric lobes. These are the regions of space tailward of the polar cap delimited by the mantle and the plasmasheet. It is usually assumed that open field lines prevail in the lobes. The Cluster orbit transverse these regions during several hours each orbit from July to October.

The lobes are also noted for their notoriously tenuous plasma, making accurate density measurements difficult. This is due to the high charging levels of the spacecraft attained here and the cold plasma populations encountered. However, using the technique described in Pedersen et al. (2008) such problems can be overcome. This method relies on measurements of the spacecraft potential, and, through a proper calibration procedure, relates these values to the ambient plasma density.

Even though the Cluster satellites are equipped with ion emitters (see Riedler et al., 1997) it is usually not possible to bring the spacecraft potential to low enough values to enable reliable measurements of the plasma density with traditional methods in the lobes. In addition, the ion emitters are usually only operated every second orbit and even less frequently as the mission progressed. Hence, the spacecraft potential method yields the most accurate and readily available plasma density measurements throughout this scantily mapped region.

The high spacecraft potentials encountered in the lobe regions was established early on by e.g. Lindqvist (1983), who used ISEE-1 data to show that positive potentials in the

Published by Copernicus Publications on behalf of the European Geosciences Union. 
region of $20-50 \mathrm{~V}$ were regularly obtained. Such observations have since been confirmed and extended by e.g. Laakso (2002), who even observed spacecraft potentials in the region $50-70 \mathrm{~V}$ on the Polar satellite. Similar measurements have also been obtained by Cluster as reported by Pedersen et al. (2008).

Previously, general surveys of magnetospheric densities, based on the spacecraft potential method, have been carried out by using data from both ISEE-1 (see Escoubet et al., 1997b) and Polar (see Laakso et al., 2002a, b). These have already established plasma densities of the order of $0.1 \mathrm{~cm}^{-3}$ for the magnetospheric lobe regions. In the present paper we will focus more on the lobes, studying them during different solar wind conditions and magnetospheric activity.

In the next two sections we will outline the spacecraft potential method itself as well as the selection criteria for our data base. Then there follows a discussion chapter and finally a summary of results.

\section{Method}

The spacecraft potential method for measuring plasma density builds on a fit between the measured spacecraft potential and the ambient plasma density obtained through calibration. The method was initially established by Knott et al. (1983), and by Schmidt and Pedersen (1987), based on observations from geostationary orbit. Later on the method was significantly extended by Pedersen (1995) and Escoubet et al. (1997b) using data from several more plasma regions. Consequently, this method has now been established as robust enough to enable the kind of magnetospheric surveys as mentioned previously (see Escoubet et al., 1997b; Laakso et al., 2002a, b).

On Cluster the spacecraft potential measurements are obtained as part of the regular operation of the Electric Field and Wave (EFW) experiment. This consists of four spherical probes and preamplifiers located at the tips of radial wire booms mounted on each spacecraft. The booms are spinning along with the spacecraft at $0.25 \mathrm{~Hz}$, and the static electric field measurements are obtained by measuring the potential difference between pair of probes using the $88 \mathrm{~m}$ baseline provided by this configuration. Time varying fields are measured according to the particular time resolution employed. A more detailed description of the EFW instrument and measurement methods are given in Gustafsson et al. (1997).

In general, any conductive surface immersed in a plasma will attain a potential relative to the surrounding plasma such that the sum of currents to it becomes zero. This equilibrium situation will be attained essentially instantaneously in a particular environment, but the vehicle potential may change as a function of time as the spacecraft moves through various regions of space. Theoretically, there could be a large number of contributing current sources including various thermal particle populations, any locally occurring energetic beams, photo-electrons as well as active current collection or emission from the vehicle itself.

However, a satellite in the tenuous lobe plasma region will attain an equilibrium potential where collected ambient electrons and escaping photo-electrons balance each other. In this plasma ion currents are negligible in comparison. With knowledge of the photo-electron escape current as a function of spacecraft potential it is then possible to estimate the electron density leading to the equilibrium current. Typical satellite potentials in the lobes are in the range 30-50 V. Solar radiation in the EUV range are required to generate photoelectrons with enough energy to escape a satellite at these large positive potential values.

In such a tenuous plasma, the potential attained by the spacecraft will consequently be so high that most of the ion population will be excluded from the ion sensors yielding ion density measurements in this region of little value. The electron spectra on the other hand may be contaminated by photo-electrons. Even active sounder techniques are often inaccurate in this environment due to low plasma density and high photo-electron flux. However, since the spacecraft potential will be a function of the properties of the surrounding plasma a proper calibration of these measurements will yield good estimates of the ambient plasma density with a high time resolution.

The spacecraft potential is determined by measuring the potential difference between the main body of the satellite and the much smaller electric field probes controlled to be near their local plasma potential by balancing their photoelectron current with a current from a high impedance source on the spacecraft itself. The potential distribution around Cluster in a tenuous plasma has been modeled by Cully et al. (2007), who showed that plasma potential near the probes located $44 \mathrm{~m}$ from the spacecraft is approximately $20 \%$ of the spaceraft potential relative to the ambient plasma potential.

By establishing a functional dependence between the spacecraft potential and the ambient plasma density through a thorough calibration program, the spacecraft potential measurements may then be routinely converted to density measurements. A detailed explanation of this calibration procedure is given in Pedersen et al. (2008). The relationship thus obtained is given by the equation:

$N_{e}(\mathrm{EFW})=A e^{-V_{s p} / B}+C e^{-V_{s p} / D}\left[\mathrm{~cm}^{-3}\right]$

where the coefficients $A, B, C$ and $D$ in principle are varying over the solar cycle. Here, $V_{s p}$ (given in units of Volt) is the potential difference between the spacecraft and the probes, and as such represent the direct measurements. From the above equation it can be seen that $B$ and $D$ are given in units of Volt while $A$ and $C$ are given in $\mathrm{cm}^{-3}$.

As explained in Pedersen et al. (2008), it is only possible to describe the variation of the coefficients $A$ and $B$ in the first tem in Eq. (1) over the solar cycle. The yearly values for these are given in Table 1. 
Table 1. This table shows the variations of the coefficients $A$ and $B$, as defined in Eq. (1), over the waning phase of solar cycle 23 . These values are taken from Pedersen et al. (2008).

\begin{tabular}{cccccccc}
\hline & 2001 & 2002 & 2003 & 2004 & 2005 & 2006 & 2007 \\
\hline$A$ & 10.0 & 6.0 & 4.0 & 3.0 & 2.3 & 2.0 & 2.0 \\
$B$ & 7.4 & 8.2 & 8.6 & 8.8 & 9.0 & 9.0 & 9.0 \\
\hline
\end{tabular}

However, an estimate of the high energy photo-electrons escaping from the spacecraft for $V_{s p}>+40 \mathrm{~V}$ could only be carried out in 2004. Separation between Cluster spacecraft was then small, and comparisons between one spacecraft being driven more positive by high electron emission from the Electron Drift Instrument (EDI) and the others without EDI high current could then be used for this estimate. We are consequently unable to find the variation in density estimates for 2001-2007 for $V_{s p}>+40 \mathrm{~V}$. Due to this lack of calibrations at very high potentials the coefficients $C$ and $D$ in the second term can only be described as being constant throughout the entire mission. Their values are $C=0.05 \mathrm{~cm}^{-3}$ and $D=30 \mathrm{~V}$. Hence, the second term can in reality be viewed as a correction which is only important at very high spacecraft potentials.

It should be noted that Eq. (1) is not valid during time periods when the ion emitters are operating. Furthermore, EFW measurements are only valid at positive spacecraft potentials above $10 \mathrm{~V}$ and cannot exceed about $70 \mathrm{~V}$ to avoid any saturation problems. Hence, from Eq. (1) it can be established that this method are applicable in the density regime $0.001-$ $1 \mathrm{~cm}^{-3}$. It should also be noted that the conversion is totally dominated by the second term of Eq. (1) for spacecraft potentials above about $50 \mathrm{~V}$.

The variation of Eq. (1) across the waning phase of the solar cycle is illustrated in Fig. 1. Here Eq. (1) is plotted using the values for coefficient $A$ and $B$ given in Table 1 . The diminishing values obtained for the density at a particular spacecraft potential is just an expression for the decrease of the photo-electric current throughout the waning phase of the solar cycle. This change may induce a factor 3-4 in estimated plasma density. Referring to calibrations in Pedersen et al. (2008) it is estimated that the uncertainty in the plasma density is about $5 \%$ below $40 \mathrm{~V}$ increasing to $20 \%$ at high potentials. This is reflected in Fig. 1 where e.g. the curve for 2001 crosses the others at high potentials.

\section{Data selection}

As the present objective is to describe the plasma density in the magnetospheric lobes, only measurements obtained in that region are discussed here. However, in order to extract these data from the general database a set of selection cri-

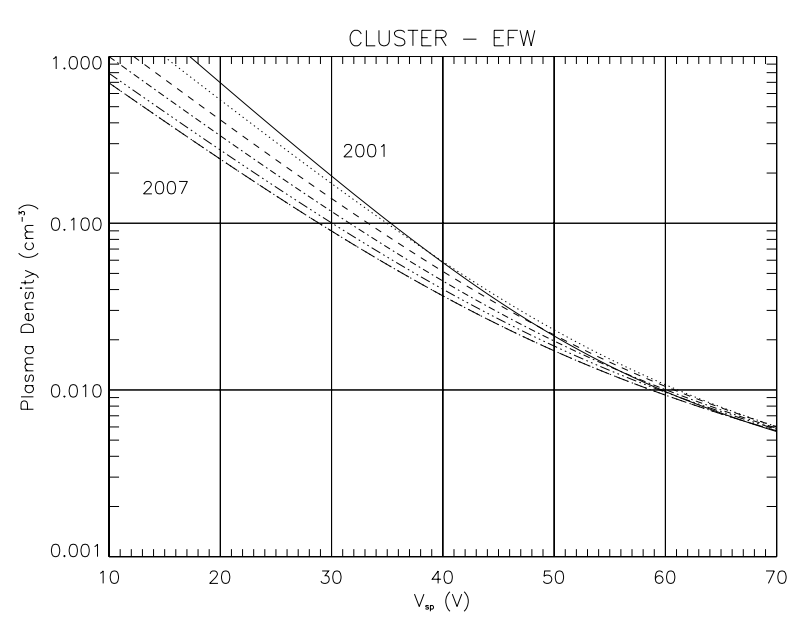

Fig. 1. This figure shows the relationship between the ambient plasma density and $V_{s p}$, the measured spacecraft to probe potential difference as given by Eq. (1). The various curves are obtained by using the coefficients given in Table 1 . The differences between the curves are due to the diminishing photo-electric current throughout the waning phase of the solar cycle.

teria has been utilized. First of all, only data from the period July, August, September and October from the years 2001-2007 have been selected. This corresponds to data coverage of $\pm 60^{\circ}$ from the GSM XZ-plane. Furthermore, an operational definition of the lobes as the region of space where $-5 R_{E} \geq X_{\mathrm{GSM}} \geq-20 R_{E},+15 R_{E} \geq Y_{\mathrm{GSM}} \geq-15 R_{E}$ and $\left|Z_{\mathrm{GSM}}\right| \geq 3 R_{E}$ has been employed. This is, as far as possible, to avoid the parts of the orbit which crossed the polar cap and the plasmasheet.

In an effort to improve on the quality of this database it has also been decided to discard all cases where the density was more than $0.5 \mathrm{~cm}^{-3}$ or the magnetic field strength was less than $20 \mathrm{nT}$ as measured by the Cluster magnetometer (see Balogh et al., 1997). The latter two criteria are used in an attempt to avoid periods of intrusion into the plasmasheet, which also may occur in this part of space.

Additionally, only data from S/C-4 are utilized since the Electron Drift Instrument (EDI), see Paschmann et al. (1997), was not operating on this spacecraft. This avoids the problem of periods of EDI high beam current operation. Additionally, all data from periods where the Active Spacecraft Potential Control (ASPOC), see Riedler et al. (1997), was operating have been discarded. Finally, the short periods where the Waves of High frequency and Sounder for Probing Electron density by Relaxation experiment (WHISPER), see Décréau et al. (1997), was transmitting within these intervals were also left out.

Since the spacecraft potential is expected to be fairly high in this region, any measurements above $+68.5 \mathrm{~V}$ (slightly less than the instrument limit) are disregarded so as to avoid saturation problems. In order to obtain the final data set the electron density is computed from each potential measurement 

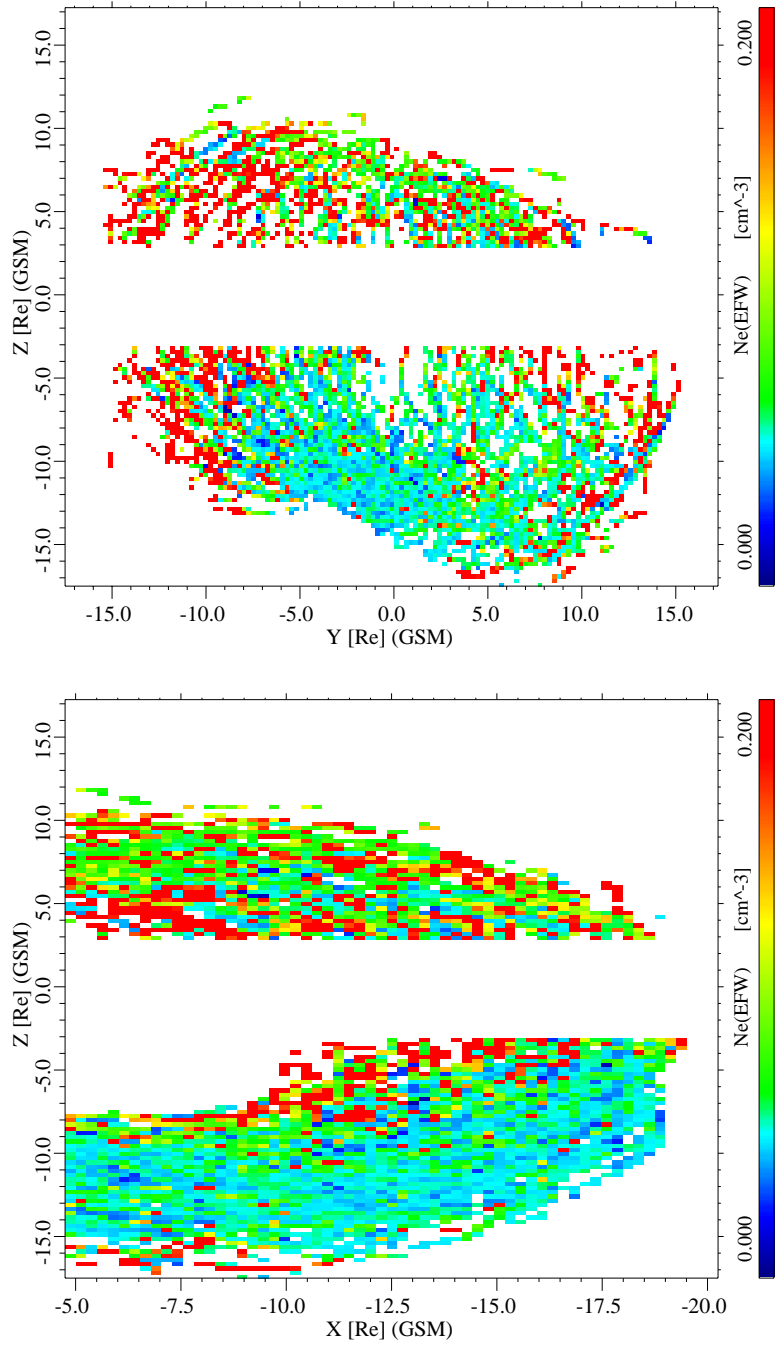

Fig. 2. This figure shows a color coded representation of the entire data space. In the top part the view is from the sun into the $\mathrm{ZY}$-plane, and the bottom view is from the evening side into the ZX-plane. In each of these two plots the measurements are collapsed into two planes with every square representing the most likely density measurement along the axis perpendicular to the particular plane.

and then averaged over $64 \mathrm{~s}$ to match the time resolution of the solar wind data. This yielded 376731 data points altogether. This database represents one of the most consistent density data set available describing the magnetospheric lobes.

The corresponding solar wind data were obtained from instruments on the ACE-satellite, orbiting the L1-point. Interplanetary magnetic field data were taken from the MAGinstrument (see Smith et al., 1998), and the plasma data from the SWEPAM-instrument (see McComas et al., 1998) both at $64 \mathrm{~s}$ time resolution. Consequently, this will be the standard time resolution used throughout the ensuing analysis.
The solar wind delay time is then taken to be the distance from the measurement point to the subsolar magnetopause, assumed to be at $10 R_{E}$, divided by the propagation velocity at the measurement point. This simple method has been shown to be adequate when using ACE data since this spacecraft is located fairly close to the Sun-Earth line (see Mailyan et al., 2008). However, in order to account for residual uncertainties in solar wind delay time as well as the response time of the magnetosphere to changes in the external conditions, an instability criterion for the interplanetary field conditions has been utilized. This criterion follows the method introduced by Haaland et al. (2007) for their convection analysis of EDI-data.

For the Interplanetary Magnetic Field (IMF) a bias vector $\boldsymbol{b}$ is constructed from the set of individual magnetic field vectors by normalizing, adding and averaging using the entire set. The angle between this vector and the GSM-Z axis then defines the clock angle. The magnitude of this bias vector then represents a measure of the stability of the IMF at the magnetopause (if the IMF was perfectly stable throughout the averaging period the magnitude of $\boldsymbol{b}$ would be exactly 1).

For this study the bias vector was constructed from measurements covering a time interval of $20 \mathrm{~min}$ before and 20 min after a particular density measurement. This time interval accounts both for any remaining uncertainties in the solar wind delay as well as additional time needed to propagate the external forcing to the lobe region. The stability criterion for IMF is taken to be a length of more than 0.96 for the bias vector following the experience of Haaland et al. (2007). This reduces the data set to 123639 points altogether.

The values of the AE-index were obtained from the World Data Center for Geomagnetism in Kyoto. As usual this data set has a time resolution of one minute. Finally, fluxes of $10.7 \mathrm{~cm}$ solar radiation were obtained from the NOAA National Geophysics Data Center. This parameter will serve as a solar cycle indicator.

In order to be able to work quantitatively with data from these various sources it was necessary to resample all observations on to a common timeline. Naturally, the Clustermeasurements formed the basis for this operation. This set then provided a consistent database for further analysis.

\section{Discussion}

The data set is characterized by a fairly large spread in density values, covering about two orders of magnitude. Actual measurements are distributed throughout the range $0.007 \mathrm{~cm}^{-3}$ to $0.5 \mathrm{~cm}^{-3}$ with a strong preference for the lower part. It should also be noted that the high value is of course limited by the filtering criteria defining the data set.

A color coded representation of the entire data set is displayed in Fig. 2, giving an averaged location of the observations. In each of these two plots the measurements are 


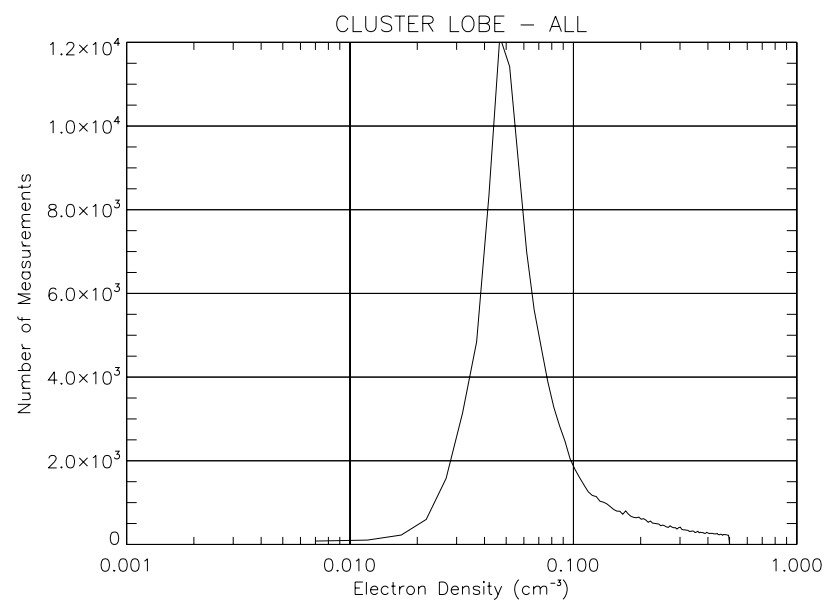

Fig. 3. This figure shows the distribution of the number of measurements as a function of electron density. The plot displays the resulting histogram after dividing the full density range into 100 bins. As is seen from the sharp maximum, the most likely density encountered here is $0.047 \mathrm{~cm}^{-3}$.

collapsed into two planes with every square representing the most likely density measurement along the axis perpendicular to the particular plane. That is, the observation space has been divided into elongated rectangular regions of sides $0.25 R_{E}$ in the view plane and $15 R_{E}$ along the $\mathrm{X}$-axis in Fig. 2a as well as $30 R_{E}$ along the Y-axis in Fig. 2b (GSMcoordinates).

The most likely density measurement found inside each such region has than been plotted with a view from the sun into the ZY-plane in the top figure and from the evening side into the ZX-plane in the bottom figure. The color code is a linear representation of the density in each region according to the color bar at the right of the figure with red being the highest density and blue the lowest. From the location of these data points it may be inferred that it is unlikely that measurements from the plasmasheet crossings have been included in the data base. It should be noted that the regions of high plasma density (red) is spread throughout the lobes and as such seems to be an inherent feature of the region. This reflects the variable nature of the plasma in the region, and it underlines the importance of high spatial resolution of the measurements.

From Fig. 2a there seems to be a surplus of high density measurements along the flanks. This could be an indication of a certain interaction along the magnetopause boundary, maybe through turbulent diffusion. It is important to realize though that this figure shows only a small gradient towards the plasmasheet. The high densities apparently shown towards the tail in Fig. $2 b$ are actually observations obtained towards the flanks. Instead the data suggests a small density decrease downtail, indicating a source region closer to the Earth.
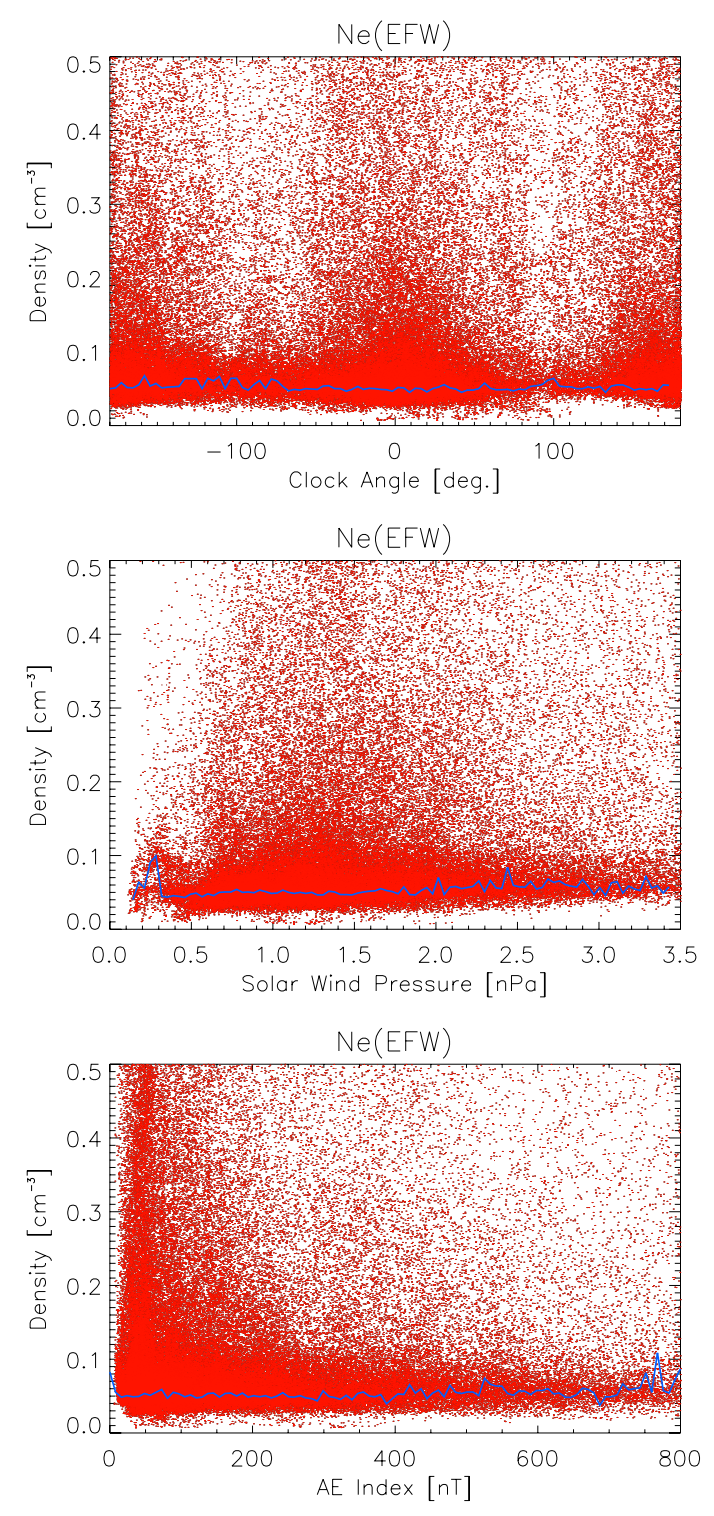

Fig. 4. This figure shows the electron density plotted as function of clock angle of the interplanetary magnetic field (top), the electron density plotted as function of solar wind pressure (middle) and the electron density plotted as function of the AE-index (bottom). Each plot also contains a blue line giving the most likely density measurement at each value of the free variable parameter in order to accommodate for any uneven distribution of density measurements.

There is also indicated a density gradient from the northern to the southern lobe, which are evident in both parts of the figure. However, it is important to note that all these gradients are small, maybe $20 \%$ or less. Thus, the overall impression is of the magnetospheric lobes as a fairly homogeneous region of space. This apparent bias towards a surplus of measurements in the southern lobe is just the natural consequence of the development of the orbit throughout the mission period. The most likely measurement value is used in 


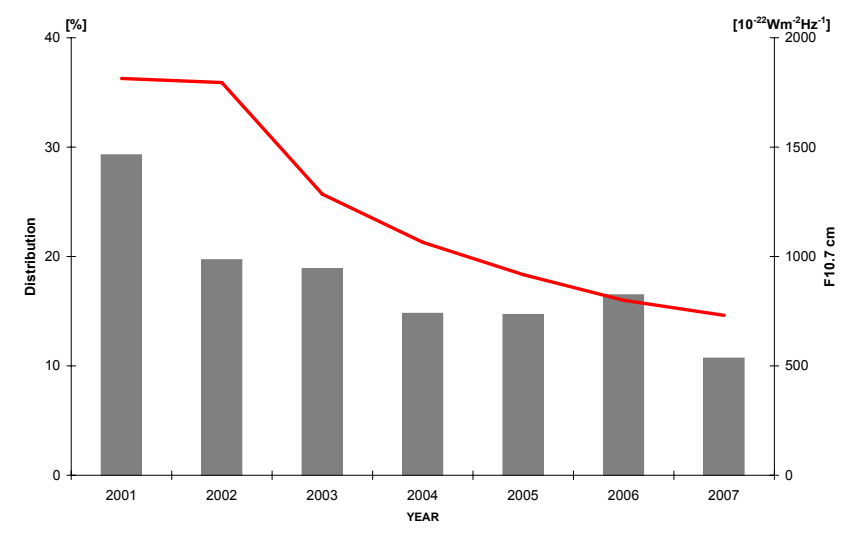

Fig. 5. This figure shows the yearly density histogram of only those measurements which are higher than $0.2 \mathrm{~cm}^{-3}$. This is overlaid by the red line showing the waning curve of the $10.7 \mathrm{~cm}$ solar radio flux. A diminishing trend is clearly seen throughout this period which also constitutes the waning part of solar cycle 23 .

this paper instead of the mean value in order to emphasis that the vast majority of observations show a very low density in this region.

This is further illustrated in Fig. 3 where a histogram of the entire data set is displayed, showing the strong probability of obtaining measurements less than $0.1 \mathrm{~cm}^{-3}$ in this region as previously reported by e.g. Escoubet et al. (1997b). Here the density range is divided into 100 bins and the number of measurements within each bin is plotted. The most likely density value found from this estimate is $0.047 \mathrm{~cm}^{-3}$, and fully two thirds of the measurements are found within the density range $0.007 \mathrm{~cm}^{-3}$ to $0.092 \mathrm{~cm}^{-3}$.

In order to assess likely source regions for this population, a correlation analysis has been carried out using the Pearson product-moment method (Moore, 2006). Processes representing source regions both internal and external to the magnetosphere have been investigated. Plots of the various parameters sets are given in Fig. 4. These shows the density plotted as function of clock angle of the interplanetary magnetic field (top), the density plotted as function of solar wind pressure (middle) and density plotted as function of the AEindex (bottom). Each plot also contains a blue line giving the most likely density measurement at each value of the free variable parameter in order to accommodate for any uneven distribution of density measurements.

Even from the plots presented in Fig. 4, it is clear that no significant correlation exists between these parameters. First of all direct entry from the solar wind seem to be ruled out by noting that the linear correlation coefficients between the lobe density and the clock angle of the interplanetary magnetic field is very small (the exact value being 0.052). As for the correlation with the solar wind pressure this coefficient is larger (with an exact value 0.140), but this is still not significant. Rather, it probably points towards a small effect on the density from the compression of the magnetopause.
Hence, both of these results points toward a dominating internal plasma source. This is seemingly at odds with previous observations carried out further downtail. Even from early observations at lunar orbit, see e.g. Hardy et al. (1976), or at larger distances, see e.g. Gosling et al. (1985), a clear correlation with the interplanetary magnetic field was established. Later on such results have been confirmed and further expanded on using observations from the Geotail-mission. In fact, it now seems clear that the distant magnetotail plasma consists of a mixture of mantle and plasmasheet populations (see e.g. Maezawa and Hori, 1998).

However, it is reasonable to assume that since these measurements were obtained at comparatively large distances downtail sufficient opportunities of interactions between the solar wind and the magnetosphere existed along this path as compared to the rather limited region of space included in our study. Hence, it is not surprising that fairly close to the Earth we find a more distinct separation between the various plasma populations as suggested by the current data base. Therefore, our conclusion of a dominating internal plasma source for our observations can be reconciled in a consistent manner with such far tail observations.

Looking further along this line of enquiry though, it must be pointed out that the linear correlation coefficient between the plasma density and the AE-index is completely negligible (the exact value being 0.006). Hence, geomagnetic activity at high latitudes, which presumably couples magnetically to the magnetospheric tail lobes, seems to have little influence on the plasma density encountered here. This suggests that the near Earth tail lobes are regions of near equilibrium between source and loss process.

In this connection it is reasonable to think of the quiet time polar wind as likely steady state source, and a continuous plasma escape downtail as a likely loss mechanism. This simple picture is collaborated by the interesting fact that the number of measurements of high densities is declining yearly as the solar cycle is waning. This is illustrated in Fig. 5, which shows the yearly density histogram of only those measurements which are higher than $0.2 \mathrm{~cm}^{-3}$. This is overlaid by the red line showing the waning curve of the $10.7 \mathrm{~cm}$ solar radio flux. A clear diminishing trend in these high density measurements is seen throughout the period.

Altogether, these measurements constitute about $14 \%$ of the entire data set. Such a clear signature in a significant part of the observations is clearly indicative of changes in the source region dependent on the solar cycle. Since variations in the geomagnetic activity have already been ruled out as a cause in this case, we are left with looking at the effects of changes due to variations in the UV/EUV part of the solar spectrum. In e.g. Cannata and Gombosi (1989) it is shown that the intensity of the polar wind is indeed likely to diminish towards the end of the solar cycle due to the strong decrease of $\mathrm{O}^{+}$-outflow. Hence, a diminishing replenishment of plasma in this region by the polar wind would result throughout our observation period. Unless the loss 
mechanisms change proportionally, this should in turn lead to far fewer cases of high density measurements at solar minimum as observed.

\section{Conclusion}

We have taken advantage of the unique orbit of the Clustermission to define a data set covering the near-Earth magnetospheric lobes. Throughout the first seven years of the mission the satellites were crossing this region of space regularly for about four months from July to October. The core of this data set consists of plasma density estimates obtained on the basis of spacecraft potential measurements from the EFWinstrument. The plasma density measurements are converted from the potential measurements using a functional relationship between these two parameters. This procedure has been described by Pedersen et al. (2008) who also discussed extensively the necessary calibration work. As pointed out in that paper the nature of this method ensures an increased accuracy of the density measurements as compared to other methods in such cold and tenuous plasma populations.

The main findings consist of a more accurate estimate of the plasma density in this region. Previous observations have indicated a density regime of less than $0.1 \mathrm{~cm}^{-3}$ in this region. Our observations have shown that throughout this period a full two thirds of the measurements were contained in the range $0.007-0.092 \mathrm{~cm}^{-3}$ irrespective of solar wind conditions or geomagnetic activity. In fact, the most probable density encountered was $0.047 \mathrm{~cm}^{-3}$, staying roughly constant throughout the entire observation period.

Furthermore, the plasma population in the region seemed to reflect an equilibrium situation in which the density is independent of the current solar wind condition or geomagnetic activity. However, the decrease of the high density tail of the population $\left(n_{e}>0.2 \mathrm{~cm}^{-3}\right)$ with the waning solar cycle points to a source region influenced by the diminishing the solar UV/EUV-intensity. Noting that the quiet time polar wind has just such a development and that it is magnetically coupled to the lobes, it seems likely to assume that this is indeed the dominating source.

It should also be noted that the method employed here provides a useful complement to more traditional ways of measuring density in that it can accomplish accurate results in plasma regimes which would otherwise not be as readily assessable. In addition, due to the high time resolution available, this method should also be useful for investigation boundary layer crossings.

Acknowledgements. This work was supported by a grant from the Norwegian Research Council. Data were obtained both from the Cluster Science Data System as well as the ACE Science Center in the case of the solar wind parameters. The World Data Center for Geomagnetism in Kyoto provided the AE-index.

Topical Editor R. Nakamura thanks A. Masson and another anonymous referee for their help in evaluating this paper.

\section{References}

Balogh, A., Dunlop, M. W., Cowley, S. W. H., Southwood, D. J., Thomlinson, J. G., Glassmeier, K. H., Musmann, G., Lühr, H., Buchert, S., Acuña, M. H., Fairfield, D. H., Slavin, J. A., Riedler, W., Schwingeshcuh, K., and Kivelson, M. G.: The Cluster Magnetic Field Investigation, Space Sci. Rev., 79, 65-91, 1997.

Cannata, R. W. and Gombosi, T. I.: Modelling the solar cycle dependence of quiet-time ion upwelling at high geomagnetic latitudes, Geophys. Res. Lett., 16, 1141-1144, 1989.

Cully, C. M., Ergun, R. E., and Eriksson, A. I.: Electrostatic structure around spacecraft in tenuous plasmas, J. Geophys. Res., 112, A09211, doi:10.1029/2007JA012269, 2007.

Escoubet, C. P., Schmidt, R., and Goldstein, M.: Cluster - Science and Mission Overview, Space Sci. Rev., 79, 11-32, 1997a.

Escoubet, C. P., Pedersen, A. Schmidt, R., and Lindqvist, P.-A.: Density in the magnetosphere inferred from ISSE 1 spacecraft potential, J. Geophys. Res., 102, 17 595-17 609, 1997b.

Décréau, P. M. E., Fergeau, D., Krannoselskikh, V., Lévéque, M., Martin, P., Randriamboarison, O., Sené, F. X., Trotignon, J. G., Canu, P., and Mögensen, P. B.: Whisper, A Resonance Sounder and Wave Analyzer: Performances and Perspectives for the Cluster mission, Space Sci. Rev., 79, 157-193, 1997.

Gosling, J. T., Baker, D. N., Bame, S. J., Feldman, W. C., and Zwickl, R. D.: North-South and Dawn-Dusk Plasma asymmetries in the in the Distant Tail Lobes: ISEE-3, J. Geophys. Res., 90, 6354-6360, 1985.

Gustafsson, G., Bostrom, R., Holmgren, G., Lundgren, A., Stasiewicz, K., Ahlen, L., Mozer, F. S., Pankow, D., Harvey, P., Berg, P., Ulrich, R., Pedersen, A., Schmidt, R., Butler, A., Fransen, A., Klinge, D., Fälthammar, C.-G., Linqvist, P.-A., Christenson, S., Holtet, J., Lybekk, B., Sten, T. A., Tanskanen, P., Lappalainen, K., and Wygant, J.: The Electric Field and Wave Experiment for the Cluster mission, Space Sci. Rev., 79, 137156, 1997.

Haaland, S. E., Paschmann, G., Förster, M., Quinn, J. M., Torbert, R. B., McIlwain, C. E., Vaith, H., Puhl-Quinn, P. A., and Kletzing, C. A.: High-latitude plasma convection from Cluster EDI measurements: method and IMF-dependence, Ann. Geophys., 25, 239-253, 2007, http://www.ann-geophys.net/25/239/2007/.

Hardy, D. A., Freeman, J. W., and Hills, H. K.: Plasma observations in the magnetotail, Magnetospheric Particles and Fields, edited by: McCormac, B. M., pp. 89-98, 1976.

Knott, K., Decreau, P. M. E., Korth, A., and Pedersen, A.: Observations of the GEOS equilibrium potential and its relation to the ambient electron energy distribution, in: Spacecraft Plasma Interactions and Their Influence on Field and Particle Measurements, Proceedings of the 17th ESLAB Symposium, ESA SP198, pp. 19-24, 1983.

Laakso, H.: Variation of the spacecraft potential in the magnetosphere, J. Atmos. Solar-Terr. Phys., 64, 1735-1744, 2002.

Laakso, H., Pfaff, R., and Janhunen, P.: Polar observations of electron density distribution in the Earth's magnetosphere. 1. Statistical results, Ann. Geophys., 20, 1711-1724, 2002a, http://www.ann-geophys.net/20/1711/2002/.

Laakso, H., Pfaff, R., and Janhunen, P.: Polar observations of electron density distribution in the Earth's magnetosphere. 2. Density profiles, Ann. Geophys., 20, 1725-1735, 2002b, http://www.ann-geophys.net/20/1725/2002/. 
Lindqvist, P.-A.: The Potential of ISEE in Different Plasma Environments, in: Spacecraft Plasma Interactions and Their Influence on Field and Particle Measurements, Proceedings of the 17th ESLAB Symposium, ESA SP-198, pp. 25-33, 1983.

Maezawa, K. and Hori, T.: The distant magnetotail: Its structure, IMF dependence, and thermal properties, New Perspectives on the Earth's Magnetotail, edited by: Nishida, A., Baker, D. N., and Cowley, W. H., pp. 1-19, 1998.

Mailyan, B., Munteanu, C., and Haaland, S.: What is the best method to calculate the solar wind propagation delay?, Ann. Geophys., 26, 2383-2394, 2008, http://www.ann-geophys.net/26/2383/2008/.

McComas, D. J., Bame, S. J., Barker, P., Feldman, W. C., Phillips, J. L., Riley, P., and Griffee, J. W.: Solar Wind Electron Proton Alpha Monitor (SSWEPAM) for the Advanced Composition Explorer, Space Sci. Rev., 86, 563-612, 1998.

Moore, D.: Basic Practice of Statistics, WH Freeman Company, ISBN: 0-7167-7463-1, 2006.

Paschmann, G., Melzner, F., Frenzel, R., Vaith, H., Parigger, P., Pagel, U., Bauer, O. H., Haerendel, G., Baumjohann, W., Scopke, N., Torbert, R. B., Briggs, B., Chan, J., Lynch, K., Morey, K., Quinn, J. M., Simpson, D., Young, C., McIlwain, C. E., Fillius, W., Kerr, S. S., Mahieu, R., and Whipple, E. C.: The Electron Drift Instrument for Cluster, Space Sci. Rev., 79, 233-269, 1997.
Pedersen, A.: Solar wind and magnetosphere plasma diagnostics by spacecraft electrostatic potential measurements, Ann. Geophys., 13, 118-129, 1995, http://www.ann-geophys.net/13/118/1995/.

Pedersen, A., Lybekk, B., Andre, M., Eriksson, A., Masson, A., Mozer, F., Lindqvist, P.-A., Decreau, P. M. E., Dandouras, I., Sauvaud, J.-A., Fazakerley, A., Taylor, M., Paschmann, G., Svenes, K. R., Torkar, K., and Whipple, E.: Electron density estimations from spacecraft potential measurements on Cluster in tenuous plasma regions, J. Geophys. Res., 113, A07S33, doi:10.1029/2007JA012636, 2008.

Riedler, W., Torkar, K., Rüdenauer, F., Fehringer, M., Pedersen, A., Schmidt, R., Grard, R. J. L., Arends, H., Narheim, B. T., Trøim, J., Torbert, R., Olsen, R. C., Whipple, E., Goldstein, R., Valavanoglou, N., and Zhao, H.: Active spacecraft potential control, Planet. Space Sci., 79, 271-302, 1997.

Schmidt, R. and Pedersen, A.: Long-term behaviour of photoelectron emission from the electric field double probe sensors on GEOS-2, Planet. Space Sci., 35, 61-67, 1987.

Smith, C. W., L'Heureux, J., Ness, N. F., Acuña, M. H., Burlaga, L. F., and Scheifele, J.: The ACE Magnetic Fields, Experiment, Space Sci. Rev., 86, 613-632, 1998. 